\title{
Resilience and Work-Related Stress May Affect Depressive Symptoms in Nursing Professionals during the COVID-19 Pandemic Era
}

\author{
Youjin Hong ${ }^{1}$, Jukab Lee ${ }^{2}$, Hyuk Joo Lee ${ }^{2}$, Kyumin Kim³, Inn-Kyu Cho ${ }^{3}$, \\ Myung Hee Ahn ${ }^{4}$, Yong-Wook Shin ${ }^{3}$, Jangho Park ${ }^{2}$, and Seockhoon Chung ${ }^{3} \bowtie$ \\ 1Department of Psychiatry, GangNeung Asan Hospital, University of Ulsan College of Medicine, Gangneung, Republic of Korea \\ ${ }^{2}$ Department of Psychiatry, Ulsan University Hospital, University of Ulsan College of Medicine, Ulsan, Republic of Korea \\ ${ }^{3}$ Department of Psychiatry, Asan Medical Center, University of Ulsan College of Medicine, Seoul, Republic of Korea \\ ${ }^{4}$ Division of Psychiatry, Health Screening and Promotion Center, Asan Medical Center, Seoul, Republic of Korea
}

Objective We aimed to investigate the effect of nursing professionals' resilience on their mental health, work-related stress, and anxiety in response to the COVID-19 pandemic.

Methods We conducted an online survey in the Asan Medical Center and Ulsan University Hospital, South Korea. We extracted data of 824 nursing professionals who consented to participate, including demographic variables and the Stress and Anxiety to Viral Epidemics-9 (SAVE-9), PHQ-9, GAD-7, and Brief Resilience Scale scores.

Results Resilience was negatively correlated with Patient Health Questionnaire-9 (PHQ-9) (rho=-0.23), Generalized Anxiety Scale-7 items (GAD-7) (rho=-0.25), Stress and Anxiety to Viral Epidem-ics-6 items (SAVE-6) (rho=-0.15), and Stress And anxiety to Viral Epidemics-3 items (SAVE-3) (rho=-0.13, all, $\mathrm{p}<0.001)$. Logistic regression analysis adjusting age, marital status, and years of employment revealed that high level of general anxiety [adjusted odds ratio $(\mathrm{aOR})=1.40,95 \%$ confidence interval $(\mathrm{CI})=1.31-1.50]$, work-related stress during viral epidemics $(\mathrm{aOR}=1.16,95 \% \mathrm{CI}=1.03-1.29)$, and a low level of resilience $(\mathrm{aOR}=0.91,95 \% \mathrm{CI}=0.85-0.97)$ were expecting variables for the depression of healthcare workers.

Conclusion Nursing professionals' level of resilience may be associated with low level of work-related stress and anxiety induced by a viral epidemic. We need to explore further the possibility of resilience as coping strategy of healthcare workers in this pandemic era.

Psychiatry Investig 2021;18(4):357-363

Key Words Resilience, Occupational stress, Health personnel, COVID-19, SAVE-9.

\section{INTRODUCTION}

Coronavirus disease 2019 (COVID-19) is a highly infectious disease caused by the severe acute respiratory syndrome coronavirus 2 (SARS-CoV-2). ${ }^{1}$ As the World Health Organization

Received: January 17, 2021 Revised: March 10, 2021

Accepted: March 14, 2021

$\triangle$ Correspondence: Jangho Park, MD, PhD

Department of Psychiatry, Ulsan University Hospital, University of Ulsan College of Medicine, 877 Bangeojinsunhwando-ro, Dong-gu, Ulsan 44033, Republic of Korea

Tel: +82-52-250-8945, Fax: +82-52-250-7078, E-mail: parkjangho@uuh.ulsan.kr

$\triangle$ Correspondence: Seockhoon Chung, MD, PhD

Department of Psychiatry, Asan Medical Center, University of Ulsan College of Medicine, 86 Olympic-ro 43-gil, Songpa-gu, Seoul 05505, Republic of Korea Tel: +82-2-3010-3411, Fax: +82-2-485-8381, E-mail: schung@amc.seoul.kr

(a) This is an Open Access article distributed under the terms of the Creative Commons Attribution Non-Commercial License (https://creativecommons.org/licenses/bync/4.0) which permits unrestricted non-commercial use, distribution, and reproduction in any medium, provided the original work is properly cited. declared COVID-19 a pandemic on March 11, 2020, ${ }^{2}$ millions of people around the world have been affected all aspects of daily life. In Korea, to government tried to prevent the spread of the disease by raising the country's infectious disease alert level to "highest" and taking strong actions such as a lockdown, social distancing, and quarantine. ${ }^{3}$ However, healthcare workers must do their job regardless of these measures. Unlike common disasters, infectious disease disasters are more difficult for the healthcare workers who care for these patients because the victims affect the hospital. Indeed, they are at high risk of being exposed to COVID-19 infection.

Previous studies from the SARS and Ebola epidemics have shown that healthcare workers can lead to severe emotional distress, anxiety, depression and posttraumatic stress disorder. ${ }^{4,5}$ Similarly, frontline healthcare workers directly involved in the diagnosis, treatment, and care of patients with COVID-19 
are at risk of developing mental health symptoms. ${ }^{6}$ Sources of distress may include increased workload, nosocomial transmission, and a fear of infecting their family, friends, or colleagues. ${ }^{7}$ Moreover, infected individuals face the fear of dying from the disease, isolation from family, and getting stigmatized by society. As a result, healthcare workers have a significantly higher prevalence of psychological problems compared to the general population during pandemics. ${ }^{8}$

Nurses have had the highest levels of occupational stress and resulting distress compared to other groups. ${ }^{9}$ Frontline nurses treating patients with COVID-19 are likely exposed to the highest risk of infection because of their close, frequent contact with patients and longer working hours. ${ }^{10}$ Nurses usually working a three-shift schedule are vulnerable to insomnia, anxiety, and depression. ${ }^{11}$ Nurses are mostly female, and the prevalence of anxiety and depression appears to be higher in females. ${ }^{12}$ Besides, the high staff turnover rate can lead to trouble with adapting to new protocols. Several reports have revealed the psychological distress of nurses during the COVID-19 pandemic. ${ }^{13,14}$ Thus, it is important to develop a stress management system for healthcare workers, especially during this pandemic.

Resilience means the capacity to recover quickly from difficulties. It is the ability to adapt positively to negative stressful events in our life. It is a complex phenomenon including personality, interpersonal relationships, and the temporal characteristics of the stressor. ${ }^{15}$ From another perspective, ${ }^{16}$ resilience is the ability to accept stress as an opportunity, e.g., recognition of limits to control, ${ }^{17}$ self-efficacy, strengthening effects of stress, adaptability to change, and sense of humor. ${ }^{18}$ It is also patience and tolerance of negative affect, and adaptability to change.

Psychologically, resilience, as an adaptive coping strategy, may be helpful to reduce healthcare workers' work-related stress and psychiatric symptoms in response to taking care of infected patients and preventing themselves from becoming infected with the virus during this pandemic. In this study, we explored the effect of resilience of nursing professionals on depression, anxiety, and work-related stress during the COVID-19 pandemic.

\section{METHODS}

\section{Participants}

An online survey was conducted in two teaching hospitals of the University of Ulsan College of Medicine, the Asan Medical Center at Seoul and Ulsan University Hospital at Ulsan, South Korea. We compiled the online survey and advertised it on the intranet of each hospital for voluntary enrollment of the healthcare workers to assess their work-related stress and anxiety response to the COVID-19 viral pandemic. From April 20 to April 30, 2020, 1,023 workers responded voluntarily to the survey and 1,019 workers from the Asan Medical Center consented to participate in the study. From 22 June to 8 July, 2020, 406 healthcare workers from the Ulsan University Hospital responded and consented to participate in the study. The protocol of each study was approved by the Institutional Review Board of Asan Medical Center (2020-0580) and Ulsan University Hospital (UUH 2020-06-021) and the requirement for written informed consent for participation was waived. We extracted data of 824 nursing professionals, 598 from 1,019 healthcare workers in Asan Medical Center and 226 from 406 healthcare workers in Ulsan University Hospital and analyzed the relationship between resilience and mental health profiles.

\section{Rating scales}

Stress and Anxiety to Viral Epidemics-9 (SAVE-9) scale

The SAVE-9 scale was developed to assess work-related stress and anxiety in response to viral epidemics. ${ }^{19}$ It consists of nine items that can be scored on a 5 -point scale ranging from 0 (never), 1 (rarely), 2 (sometimes), 3 (often), and 4 (always). ${ }^{20}$ This scale can be clustered into two factors: 1 ) anxiety about viral epidemics-6 items (SAVE-6), and 2) work-related stress associated with viral epidemics-3 items (SAVE-3). The cut-off score of the SAVE-9 was set to 21 points in parallel with Generalized Anxiety Disorder-7 items (GAD-7) $\geq 5$ denoting a mild degree of general anxiety. Satisfactory internal consistency (Cronbach's $\alpha=0.795$ ) was reported.

\section{Patient Health Questionnaire-9 (PHQ-9)}

The PHQ-9 is a self-rated, 9-item questionnaire for measuring depression symptoms. Each item is scored on a 3-point Likert scale $(0=$ not at all to $3=$ nearly every day). Scores can range from 0 to 27, with higher scores reflecting greater symptom severity $(0-4=$ minimal depression, $5-9=$ mild depression, 10-14=moderate depression, $15-19=$ moderately severe depression and $\geq 20=$ severe depression). ${ }^{21}$ In this study we defined a PHQ-9 score $\geq 10$ as clinical depression. Excellent internal reliability with Cronbach's $\alpha=0.89$ was reported.

\section{Generalized Anxiety Scale-7 items}

The GAD-7 is a self-administered 7-item questionnaire for measuring general anxiety. Each item is scored on a 3-point Likert scale $(0=$ not at all to $3=$ nearly every day). Scores can range from 0 to 21, with higher scores reflecting higher levels of anxiety. Cut-off intervals for anxiety include 0-4 (minimal), 5-9 (mild anxiety), 10-14 (moderate), and 15-21 (severe). ${ }^{22}$ The excellent internal consistency (Cronbach's $\alpha=$ 0.92 ) and good test-retest reliability (intraclass correlation= 
$0.83)$ were reported.

\section{Brief Resilience Scale (BRS)}

The BRS is a 6-item rating scale to measure subjects' resilience as a coping strategy to stress. ${ }^{23}$ The BRS assesses the ability to bounce back. Each question can be rated on a range from $1-5$, with a total score of 6-30. Good internal consistency (Cronbach's alpha ranging from $0.80-0.91$ ) and good test-retest reliability (intraclass correlation of 0.62 and 0.69 ) were reported. The Korean version of the BRS is validated and was thus used in our study. ${ }^{24}$

\section{Statistical analysis}

Statistical analyses were performed with SPSS ver. 21.0 for Windows (IBM Corp., Armonk, NY, USA). The clinical characteristics are summarized as mean \pm standard deviation. The level of significance for all analyses was defined as two-tailed $\mathrm{p}<0.05$. A Student's t-test for continuous variables and chisquare test for categorical variables were performed for between group analyses. A Spearman's correlation analysis was performed to explore the relationship between clinical variables. A logistic regression analysis was carried out to determine the variables that contribute to clinical depression (PHQ-9 score $\geq 10$ ).

\section{RESULTS}

Data from 824 nursing professionals at Asan Medical Center and Ulsan University Hospital were extracted and analyzed. Demographic characteristics and mental health profiles are shown in Table 1.

Among the participants, 152 (18.4\%) workers were rated as having clinical depression (PHQ-9 $\geq 10$ ). The proportion of junior staff and workers with past psychiatric history was significantly higher in the depressed group compared to the nondepressed group $(\mathrm{p}<0.001)$. Years of employment was significantly shorter in the depressed group than in the non-depressed group $(\mathrm{p}<0.001)$. Workers with clinical depression showed significantly higher PHQ-9, GAD-7, and SAVE-9 scores and lower BRS scores compared to the non-depressed group.

Older age was significantly correlated with low PHQ-9, GAD-7, SAVE-6, and SAVE-3 scores (all p<0.01) (Table 2). More years of employment significantly correlated with low PHQ-9, GAD-7, and SAVE-3 scores. Age and years of employment did not correlate with resilience level. PHQ-9 score positively correlated with high GAD-7, SAVE-6, and SAVE3 scores and low BRS scores. BRS score negatively correlated with PHQ-9, GAD-7, SAVE-6, and SAVE-3 scores (all p<0.01).

Among nursing professionals who were not depressed (PHQ-9 score $<10$ ), the overall results were similar (Table 3) to the results from the correlation analysis among all partici-

Table 1. Demographic characteristics of the participants $(\mathrm{N}=824)$

\begin{tabular}{|c|c|c|c|}
\hline Variables & Depressed* $(\mathrm{N}=152)$ & Not depressed $(\mathrm{N}=672)$ & $\mathrm{p}$ \\
\hline Sex (female) & $150(98.7)$ & $657(97.8)$ & 0.37 \\
\hline \multicolumn{4}{|l|}{ Age } \\
\hline Junior (20-39 years) & $126(82.9)$ & $439(65.3)$ & \multirow[t]{2}{*}{$<0.001$} \\
\hline Senior ( $40-65$ years) & $26(17.1)$ & $233(34.7)$ & \\
\hline \multicolumn{4}{|l|}{ Marital status } \\
\hline Single & $102(65.7)$ & $336(50.2)$ & \multirow[t]{3}{*}{$<0.001$} \\
\hline Married, without kids & $12(7.9)$ & $58(8.7)$ & \\
\hline Married, with kids & $37(24.5)$ & $275(41.1)$ & \\
\hline $\begin{array}{l}\text { Have you experienced or had treatment for depression, anxiety, } \\
\text { or insomnia? (yes) }\end{array}$ & $33(21.9)$ & $62(9.2)$ & $<0.001$ \\
\hline Years of employment (years) & $7.9 \pm 6.9$ & $11.4 \pm 8.9$ & $<0.001$ \\
\hline \multicolumn{4}{|l|}{ Symptom assessments } \\
\hline PHQ-9 & $13.2 \pm 3.3$ & $3.9 \pm 2.8$ & $<0.001$ \\
\hline GAD-7 & $9.0 \pm 4.2$ & $3.3 \pm 3.1$ & $<0.001$ \\
\hline SAVE-9 & $24.5 \pm 4.8$ & $20.5 \pm 5.3$ & $<0.001$ \\
\hline Anxiety subcategory (SAVE-6) & $16.8 \pm 3.6$ & $14.6 \pm 3.8$ & $<0.001$ \\
\hline Work-stress subcategory (SAVE-3) & $7.7 \pm 2.3$ & $5.9 \pm 2.2$ & $<0.001$ \\
\hline BRS & $18.0 \pm 3.9$ & $20.1 \pm 3.3$ & $<0.001$ \\
\hline
\end{tabular}

*defined as PHQ-9 score $\geq 10$. PHQ-9: Patient Health Questionnaire-9, GAD-7: Generalized Anxiety Scale-7 items, SAVE-9: Stress and Anxiety to Viral Epidemics-9 items, BRS: Brief Resilience Scale 
Table 2. Spearman's correlation coefficients of all variables in all participants $(\mathrm{N}=824)$

\begin{tabular}{|c|c|c|c|c|c|c|c|}
\hline Variables & Age & Years of employment & PHQ-9 & GAD-7 & SAVE-6 & SAVE-3 & BRS \\
\hline Age & 1.000 & & & & & & \\
\hline Years of employment & $0.88^{* *}$ & 1.000 & & & & & \\
\hline PHQ-9 & $-0.18^{* *}$ & $-0.19^{* *}$ & 1.000 & & & & \\
\hline GAD-7 & $-0.15^{* *}$ & $-0.14^{* *}$ & $0.72^{* *}$ & 1.000 & & & \\
\hline SAVE-6 & $-1.07^{* *}$ & -0.05 & $0.28^{* *}$ & $0.39^{* *}$ & 1.000 & & \\
\hline SAVE-3 & $-0.15^{* *}$ & $-0.09^{* *}$ & $0.35^{* *}$ & $0.45^{* *}$ & $0.45^{* *}$ & 1.000 & \\
\hline BRS & 0.03 & 0.05 & $-0.23^{* *}$ & $-0.25^{* *}$ & $-0.15^{* *}$ & $-0.13^{* *}$ & 1.000 \\
\hline
\end{tabular}

${ }^{* *} \mathrm{p}<0.01$. PHQ-9: Patient Health Questionnaire-9, GAD-7: Generalized Anxiety Scale-7 items, SAVE-6: Stress and Anxiety to Viral Epidemics-6 items, SAVE-3: Stress And anxiety to Viral Epidemics-3 items, BRS: Brief Resilience Scale

Table 3. Logistic regression analysis of variables that may contribute to depression* $(\mathrm{N}=824)$

\begin{tabular}{|c|c|c|c|c|}
\hline Variables & cOR $(95 \% \mathrm{CI})$ & $\mathrm{p}$ & $\mathrm{aOR}(95 \% \mathrm{CI})$ & $\mathrm{p}$ \\
\hline Age, junior (vs. senior) & $2.57(1.64-4.03)$ & $<0.0001$ & $1.23(0.51-2.99)$ & 0.64 \\
\hline Marital status, single (vs. married) & $2.06(1.42-2.99)$ & $<0.0001$ & $1.23(0.66-2.29)$ & 0.51 \\
\hline $\begin{array}{l}\text { Have you experienced or had treatment for depression, anxiety, } \\
\text { or insomnia? }\end{array}$ & $2.75(1.73-4.39)$ & $<0.0001$ & $0.57(0.30-1.07)$ & 0.08 \\
\hline Years of employment (years) & $0.95(0.93-0.97)$ & $<0.0001$ & $0.98(0.93-1.04)$ & 0.49 \\
\hline \multicolumn{5}{|l|}{ Rating scale scores } \\
\hline GAD-7 & $1.47(1.38-1.56)$ & $<0.0001$ & $1.40(1.31-1.50)$ & $<0.001$ \\
\hline SAVE-6 & $1.18(1.12-1.24)$ & $<0.0001$ & $1.02(0.95-1.09)$ & 0.58 \\
\hline SAVE-3 & $1.44(1.32-1.57)$ & $<0.0001$ & $1.16(1.03-1.29)$ & 0.01 \\
\hline BRS & $0.84(0.80-0.89)$ & $<0.0001$ & $0.91(0.85-0.97)$ & 0.007 \\
\hline
\end{tabular}

*depression was defined as PHQ-9 score $\geq 10$. cOR: Crude odds ratio, aOR: adjusted odds ratio, CI: confidence interval, GAD-7: Generalized Anxiety Scale-7 items, SAVE-6: Stress and Anxiety to Viral Epidemics-6 items, SAVE-3: Stress and Anxiety to Viral Epidemics-3 items, BRS: Brief Resilience Scale

pants (Table 2).

Among the 824 participants, we explored the variables that might lead to depressive symptoms in nursing professionals by adjusting for age, sex, marital status, SAVE-6 score, and participants' past psychiatric illness history using a logistic regression analysis. The results show that depression occurred in those individuals with a high level of general anxiety [adjusted odds ratio $(\mathrm{aOR})=1.40,95 \%$ confidence interval $(\mathrm{CI})=$ 1.31-1.50] and work-related stress to viral epidemics $(\mathrm{aOR}=$ $1.16,95 \% \mathrm{CI}=1.03-1.29$ ), and a low level of resilience $(\mathrm{aOR}=$ 0.91, 95\% CI=0.85-0.97) (Table 3).

\section{DISCUSSION}

In this study, the risk for clinical depression was increased by high levels of general anxiety, work-related stress in response to a viral pandemic, and a low level of resilience.

Working in a hospital during this pandemic era is extremely stressful to healthcare workers. ${ }^{5,6,8,9}$ In particular, nurses working on the frontline treating patients with COVID-19 are exposed to the highest risk of infection. ${ }^{25}$ They may face uncertainty and the fear of being infected with COVID-19 from the patients they care for, and can also experience emotional distress at the thought of infecting their family members. Therefore, the highest level of stress was observed in the group who were working in a high-risk unit. ${ }^{26}$ These individuals do not have enough time to rest, change their gown for each patient, and are often in distressing situations where patients can be uncooperative. Therefore, stress management programs are needed for healthcare workers, especially nursing professionals, who are working on the frontline during this pandemic era. ${ }^{27}$

In this study, nursing professionals with younger age and shorter working years were found to be more depressed during the viral pandemic. Nursing professionals who have worked for fewer years may have poor coping ability because they are relatively inexperienced and less skilled in their work than nurses who have worked for a long time. Therefore, they have difficulty in adapting well to distress, such as an increased workload and infection risk, and the risk of mental problems for them increases. It is also possible that junior nursing professionals are required to perform more tasks at this time. A 
greater number of working years was also associated with lower levels of anxiety and work-related stress. Senior nursing professionals are more likely to experience less distress at work as they become more experienced and skilled at their work. This may be because nursing professionals who are less anxious and less stressed about their work remain at work for a long time.

Resilience is a term used to describe the ability to recover from negative life experiences and is an important concept in the field of stress management. Whether it should be considered a temporary state of mind, meaning it can change according to the situation, or a trait, meaning it is a stable characteristic of a person, has been under debate. ${ }^{28}$ Viewing resilience as a personality trait has been very influential; however, it is limited by the denial of the complexities of psychological principles. ${ }^{29}$ In this regard, Richardson's metatheory of resilience and resiliency defined resilience as having both trait and contextual factors. ${ }^{30}$ It is possible to identify people who are less likely to experience burnout using the trait level of resilience while understanding how they are dealing with current stressors using state resilience. In this context, the most recent consensus reached is that a combination of both state and trait factors leads to the formation of resilience in each individual.

In this study, the depressed group had a lower resilience score than the non-depressed group. As the resilience score decreased, the level of anxiety and work-related stress increased. This is consistent with other studies showing that a higher level of resilience is correlated to a lower probability of experiencing mental health issues such as depression or anxiety. ${ }^{31}$ Similar results have been found in studies that explored the association between resilience and psychological outcomes, such as stress, anxiety, depression, and fatigue, in nurses. ${ }^{32}$ In our study, resilience did not correlate with age or number of years worked, but in other studies, resilient nurses were more likely to work longer because they showed successful adaptation and an active coping style in response to stressors. ${ }^{33}$

According to previous studies on the effect of resilience among healthcare workers, resilience has been proven to play an important role in overcoming distress. Likewise, in the COVID-19 pandemic era, resilience and anxiety levels have been shown to have an inverse relationship among physicians, ${ }^{34}$ frontline nurses, ${ }^{35}$ and the total group of healthcare professionals. ${ }^{36,37}$ Moreover, a recent study suggested that among general populations, more resilient adults are experiencing a lower rate of burnout and distress during the current pandemic era, which corresponds with the previous finding that resilience has an inverse association with burnout among physicians. ${ }^{38,39}$

Considering the results of this study, we should consider setting up a psychological management program for nursing professionals who are at high risk of becoming depressed. In particular, we need to consider the experience of caring (years of employment), current individual/family support, or the place individuals work in. Psychologically, we should consider the emotional status, e.g., anxiety, depression, or work-related stress. Furthermore, it is important to help individuals improve their adaptive coping strategy, resilience, and workrelated stress levels.

The limitations of this study are as follows. First, as this study involved an online self-report survey, we could not perform face-to-face interviews with the participants. Although an online survey was necessary to prevent the spread of the virus during the pandemic, this limited our ability to obtain details of psychiatric symptoms. Second, this survey was performed in a hospital in Seoul and a hospital in Ulsan, both metropolitan cities. Therefore, we cannot generalize the results from the responses of nursing professionals of these two hospitals, although the two hospitals are the largest hospitals in each city. Third, this survey was conducted in mid-April 2020, the first wave of the pandemic; thus, the results of this study do not reflect the psychiatric symptoms of nursing professionals during the third or forthcoming waves of the COVID-19 pandemic. Last, the SAVE-9 scale used in this study was not fully validated; however, we attempted to develop and apply the scale to assess anxiety and work-related stress, especially in healthcare workers during this pandemic. The validation report of the SAVE-9 scale will be formally reported soon.

In conclusion, healthcare workers, especially nursing professionals, experience various kinds of psychiatric symptoms such as depression, anxiety, and work-related stress during the COVID-19 pandemic. It is important to manage these individuals psychologically for their severe work-related stress. In this study, we observed that resilience can reduce work-related stress and anxiety to the viral pandemic, and the resultant depressive symptoms. Hospitals and the government should develop a system for healthcare workers to improve their resilience as a coping skill.

\section{Acknowledgments}

We would like to acknowledge all the healthcare workers in Ulsan University Hospital and Asan Medical Center who voluntarily participated in our survey.

\section{Conflicts of Interest}

The authors have no potential conflicts of interest to disclose.

\section{Author Contributions}

Conceptualization: Seockhoon Chung, Yong-Wook Shin, Jangho Park. Data curation: Jukab Lee, Hyuk Joo Lee, Kyumin Kim, Inn-Kyu Cho. Formal analysis: Seockhoon Chung, Jukab Lee, Jangho Park, Youjin Hong. Funding acquisition: Seockhoon Chung. Investigations: Seockhoon Chung. Methodology: Jukab Lee, Jangho Park, Seockhoon Chung, Yong-Wook Shin. Project administration: Yong-Wook Shin. Resources: Myung Hee Ahn. Visualization: Myung Hee Ahn. Writing_original draft: Youjin Hong, Myung 
Hee Ahn. Writing_review \& editing: Jukab Lee, Jangho Park, Kyumin Kim, Inn-Kyu Cho, Seockhoon Chung, Yong-Wook Shin.

\section{ORCID iDs}

Youjin Hong

Jukab Lee

Hyuk Joo Lee

Kyumin Kim

Inn-Kyu Cho

Myung Hee Ahn

Yong-Wook Shin

Jangho Park

Seockhoon Chung https://orcid.org/0000-0002-1062-1658

https://orcid.org/0000-0001-5917-1398

https://orcid.org/0000-0002-3569-9603

https://orcid.org/0000-0003-1203-1157

https://orcid.org/0000-0002-1064-3013

https://orcid.org/0000-0002-8493-020X

https://orcid.org/0000-0002-0360-6118

https://orcid.org/0000-0001-5370-005X

https://orcid.org/0000-0002-9798-3642

\section{REFERENCES}

1. Chan JF, Yuan S, Kok KH, To KK, Chu H, Yang J, et al. A familial cluster of pneumonia associated with the 2019 novel coronavirus indicating person-to-person transmission: a study of a family cluster. Lancet 2020;395:514-523.

2. Huang C, Wang Y, Li X, Ren L, Zhao J, Hu Y, et al. Clinical features of patients infected with 2019 novel coronavirus in Wuhan, China. Lancet 2020;395:497-506.

3. Korea centers for Disease Control \& Prevention. Updates on COVID-19 in Republic of Korea, Feb 23, 2020. [Updated 2020]. Available at: http://ncov.mohw.go.kr/tcmBoardView.do?brdId=\&brdGubun=\& dataGubun $=\&$ ncvContSeq $=353064 \&$ contSeq $=353064 \&$ board id=140\&gubun=BDJ. Accessed Aug 30, 2020.

4. Xiao J, Fang M, Chen Q, He B. SARS, MERS and COVID-19 among healthcare workers: a narrative review. J Infect Public Health 2020;13: 843-848.

5. Preti E, Di Mattei V, Perego G, Ferrari F, Mazzetti M, Taranto P, et al. The psychological impact of epidemic and pandemic outbreaks on healthcare workers: rapid review of the evidence. Curr Psychiatry Rep 2020;22:43.

6. Kang L, Li Y, Hu S, Chen M, Yang C, Yang BX, et al. The mental health of medical workers in Wuhan, China dealing with the 2019 novel coronavirus. Lancet Psychiatry 2020;7:e14.

7. Li W, Yang Y, Liu ZH, Zhao YJ, Zhang Q, Zhang L, et al. Progression of Mental Health Services during the COVID-19 Outbreak in China. Int J Biol Sci 2020;16:1732-1738.

8. Ji D, Ji YJ, Duan XZ, Li WG, Sun ZQ, Song XA, et al. Prevalence of psychological symptoms among Ebola survivors and healthcare workers during the 2014-2015 Ebola outbreak in Sierra Leone: a cross-sectional study. Oncotarget 2017;8:12784-12791.

9. Nickell LA, Crighton EJ, Tracy CS, Al-Enazy H, Bolaji Y, Hanjrah S, et al. Psychosocial effects of SARS on hospital staff: survey of a large tertiary care institution. CMAJ 2004;170:793-798.

10. Shih FJ, Gau ML, Kao CC, Yang CY, Lin YS, Liao YC, et al. Dying and caring on the edge: Taiwan's surviving nurses' reflections on taking care of patients with severe acute respiratory syndrome. Appl Nurs Res 2007; 20:171-180.

11. Choi SJ, Song P, Suh S, Joo EY, Lee SI. Insomnia Symptoms and mood disturbances in shift workers with different chronotypes and working schedules. J Clin Neurol 2020;16:108-115.

12. Albert PR. Why is depression more prevalent in women? J Psychiatry Neurosci 2015;40:219-221.

13. Kang L, Ma S, Chen M, Yang J, Wang Y, Li R, et al. Impact on mental health and perceptions of psychological care among medical and nursing staff in Wuhan during the 2019 novel coronavirus disease outbreak: a cross-sectional study. Brain Behav Immun 2020;87:11-17.

14. Mo Y, Deng L, Zhang L, Lang Q, Liao C, Wang N, et al. Work stress among Chinese nurses to support Wuhan in fighting against COVID-19 epidemic. J Nurs Manag 2020;28:1002-1009.

15. Bonanno GA. Loss, trauma, and human resilience: have we underesti- mated the human capacity to thrive after extremely aversive events? Am Psychol 2004;59:20-28.

16. Connor KM, Davidson JR. Development of a new resilience scale: the Connor-Davidson Resilience Scale (CD-RISC). Depress Anxiety 2003; 18:76-82.

17. Kobasa SC. Personality and resistance to illness. Am J Community Psychol 1979;7:413-423.

18. Rutter M. Resilience in the face of adversity. Protective factors and resistance to psychiatric disorder. Br J Psychiatry 1985;147:598-611.

19. Chung S, Kim H, Ahn M, Yeo S, Lee J, Kim K, et al. Development of the Stress and Anxiety to Viral Epidemics-9 (SAVE-9) scale for assessing work-related stress and anxiety in healthcare workers in response to COVID-19. PsyArxiv 2020; June 11.

20. Stress and Anxiety to Viral Epidemics-9 items for Healthcare workers. Psychiatry, ASAN medical center. Univ of Ulsan College of Medicine. [updated 2020]. Available at: [https://www.save-vira-lepidemic.net/. Accessed 20 April, 2020.

21. Kroenke K, Spitzer RL, Williams JB. The PHQ-9: validity of a brief depression severity measure. J Gen Intern Med 2001;16:606-613.

22. Spitzer RL, Kroenke K, Williams JB, Lowe B. A brief measure for assessing generalized anxiety disorder: the GAD-7. Arch Intern Med 2006; 166:1092-1097.

23. Smith BW, Dalen J, Wiggins K, Tooley E, Christopher P, Bernard J. The brief resilience scale: assessing the ability to bounce back. Int J Behav Med 2008;15:194-200.

24. Jeon SW, Han C, Choi J, Pae CU, Chae JH, Ko YH, et al. Posttraumatic growth and resilience: assessment and clinical implications. J Korean Neuropsychiatr Assoc 2015;54:32-39.

25. Schembre D. Response to integrating gastrointestinal bleeding risk scores into clinical practice. Am J Gastroenterol 2020;115:960.

26. Chen CS, Wu HY, Yang P, Yen CF. Psychological distress of nurses in Taiwan who worked during the outbreak of SARS. Psychiatr Serv 2005; 56:76-79.

27. Marjanovic Z, Greenglass ER, Coffey S. The relevance of psychosocial variables and working conditions in predicting nurses' coping strategies during the SARS crisis: an online questionnaire survey. Int J Nurs Stud 2007;44:991-998.

28. Ye ZJ, Zhang Z, Zhang XY, Tang Y, Chen P, Liang MZ, et al. State or trait? Measuring resilience by generalisability theory in breast cancer. Eur $\mathrm{J}$ Oncol Nurs 2020;46:101727.

29. De La Rosa GM, Webb-Murphy JA, Johnston SL. Development and validation of a brief measure of psychological resilience: an adaptation of the response to stressful experiences scale. Mil Med 2016;181:202-208.

30. Richardson GE. The metatheory of resilience and resiliency. J Clin Psychol 2002;58:307-321.

31. Wermelinger Avila MP, Correa JC, Lucchetti ALG, Lucchetti G. The role of physical activity in the association between resilience and mental health in older adults. J Aging Phys Act 2018;26:248-253.

32. Sampson M, Melnyk BM, Hoying J. Intervention effects of the MINDBODYSTRONG cognitive behavioral skills building program on newly licensed registered nurses' mental health, healthy lifestyle behaviors, and job satisfaction. J Nurs Adm 2019;49:487-495.

33. Ramalisa RJ, du Plessis E, Koen MP. Increasing coping and strengthening resilience in nurses providing mental health care: Empirical qualitative research. Health SA 2018;23:1094.

34. Mosheva M, Hertz-Palmor N, Dorman Ilan S, Matalon N, Pessach IM, Afek A, et al. Anxiety, pandemic-related stress and resilience among physicians during the COVID-19 pandemic. Depress Anxiety 2020;37: 965-971.

35. Labrague LJ, De Los Santos JAA. COVID-19 anxiety among front-line nurses: predictive role of organisational support, personal resilience and social support. J Nurs Manag 2020;28:1653-1661.

36. Bozdag F, Ergun N. Psychological resilience of healthcare professionals during COVID-19 pandemic. Psychol Rep 2020:33294120965477. [Online ahead of print] 
37. Barzilay R, Moore TM, Greenberg DM, DiDomenico GE, Brown LA, White LK, et al. Resilience, COVID-19-related stress, anxiety and depression during the pandemic in a large population enriched for healthcare providers. Transl Psychiatry 2020;10:291.

38. Yildirim M, Solmaz F. COVID-19 burnout, COVID-19 stress and resil- ience: Initial psychometric properties of COVID-19 Burnout Scale. Death Stud 2020:1-9. [Online ahead of print]

39. Fox S, Lydon S, Byrne D, Madden C, Connolly F, O'Connor P. A systematic review of interventions to foster physician resilience. Postgrad Med J 2018;94:162-170. 